\title{
Parasitismo na População da Broca-do-Café Hypothenemus hampei (Ferrari) (Coleoptera: Curculionidae), pelo Parasitoide Cephalonomia stephanoderis Betrem (Hymenoptera: Bethylidae)
}

\author{
Moisés Santos de Souza ${ }^{\bowtie}$, Alexandre Almeida e Silva², \\ César Augusto Domingues Teixeira ${ }^{3}$ \& José Nilton Medeiros Costa ${ }^{3}$
}

1. Universidade Federal do Amazonas - PPG-BIONORTE, e-mail: moisesantos@gmail.com (Autor para correspondência ${ }^{\bowtie}$ ). 2. Fundação Universidade Federal de Rondônia, e-mail: alealsil@unir.br. 3. Embrapa Rondônia, e-mail: cesar.teixeira@embrapa.br, jose-nilton.costa@embrapa.br.

\section{EntomoBrasilis 7 (3): 178-182 (2014)}

Resumo. A broca-do-café, Hypothenemus hampei (Ferrari) é responsável por grandes prejuízos em plantios de café, causando perdas de produtividade aos cafeicultores e danos quantitativos e qualitativos aos grãos. O controle biológico da praga tem sido principalmente à base de fungos entomopatogênicos. Em Rondônia, Brasil, as informações acerca dos parasitoides de $H$. hampei são escassas. O parasitoide Cephalonomia stephanoderis Betrem é um inimigo natural da broca do café e já é utilizado como agente de controle biológico em países da África e da América Latina. Constatou-se a presença desse parasitoide em cafezais de Rondônia. O objetivo do presente estudo foi investigar os níveis de ocorrência e parasitismo do parasitóide em uma plantação de café no município de Ouro Preto D’Oeste, Estado de Rondônia. Foram analisados semanalmente frutos de café coletados diretamente das plantas e caídos sobre o solo, de janeiro a dezembro de 2004. Observou-se a ocorrência do parasitoide durante os meses de maior infestação da praga. Durante o período estudado, a taxa de parasitismo nos diferentes microambientes variou de 2 a $24 \%$.

Palavras-Chave: Ectoparasitoide; Ocorrência; Parasitismo; Vespa da Costa do Marfim.

Parasitism in the Population of the Coffee Berry Borer Hypothenemus hampei (Ferrari) (Coleoptera: Curculionidae) by the Parasitoid Cephalonomia stephanoderis Betrem (Hymenoptera: Bethylidae)

Abstract. The coffee berry borer, Hypothenemus hampei (Ferrari) attacks coffee plantations, greatly decreasing the production of coffee plants, causing quantitative and qualitative damage to the grains. Biological control of this pest has focused mostly on entomopathogenic fungi. In the state of Rondonia, studies on parasitism levels of the parasitoids of $H$. hampei are scarce. The parasitoid Cephalonomia stephanoderis Betrem is a natural enemy of the coffee berry borer and is already used as an agent of biological control in countries of Africa and Latin America. The objective of the present study was to investigate the occurrence and parasitism levels of the parasitoid in a coffee plantation in the municipality of Ouro Preto D'Oeste, State of Rondonia. Coffee beans collected directly from the plants and on the ground were analyzed weekly from January to December 2004. Parasitoid occurred in months of the highest pest infestation. During the studied period, parasitism rate in different microenvironments ranged from 2 to $24 \%$.

Keywords: Ectoparasitoid; Ivory Coast Wasp; Occurrence; Parasitism.

岛: m todo o mundo, em regiões onde há plantações de café, a broca-do-café é a principal praga, causando prejuízos para mais de 20 milhões de famílias de agricultores (VEGa et al. 2009; Chiu-Alvarado et al. 2010) Historicamente, a presença da broca-do-café no Brasil relaciona-se com introdução de sementes oriundas da África e de Java entre os anos de 1913 a 1924, culminando na sua disseminação por todas as zonas produtoras de café no país (LAURENTINo \& CosTA 2004). Essa praga, originária do continente africano, é um pequeno coleóptero de cor preta, considerada a praga mais severa do café devido aos resultados negativos de sua ação sobre a produção cafeeira em termos quantitativo e qualitativo (DAMON \& VALLE 2002). Causa prejuízos quantitativos, pela redução do peso dos grãos e queda de frutos, e qualitativos, através da alteração da qualidade do grão e da bebida. Os danos são causados pelas larvas, que vivem no interior do fruto de café e atacam as sementes para sua alimentação, podendo destruir os frutos parcial ou totalmente (REIs et al. 2002). Em Rondônia, há registros de altas infestações (34 a 41\%) desta praga no auge da colheita. Esses níveis são altamente comprometedores para a produtividade e qualidade do café (Costa et al. 2000).
O principal agente de controle biológico testado nas áreas de plantio do café em Rondônia é o fungo entomopatogênico Beauveria bassiana (Bals) Vuill (Costa et al 2002). Entretanto, vários artrópodes de hábitos: predador e parasitoide são relatados como inimigos naturais de insetos que atacam a cultura de café (PÉREZ-LACHAud et al. 2002; SALAZAR \& BAKer 2002). Cephalonomia stephanoderis Betrem (Hymenoptera: Bethylidae), é um dos inimigos naturais mais promissores para o controle biológico da broca-do-café, devido à sua comprovada adaptação em vários agroecossistemas cafeeiros da África e América, por sua dieta específica e a existência de metodologias apropriadas para sua criação (CARBALLO 2002).

A vespa adulta mede de 1,6 a $2 \mathrm{~mm}$ de comprimento de cor preto brilhante (CARBALlo 2002). C. stephanoderis é um parasitoide que coloca seus ovos nos estágios imaturos (larvas e pupas) de Hypothenemus hampei (Ferrari), que é seu hospedeiro específico (LACHAUd \& HARDY 2001). As fêmeas deste parasitoide também se comportam exclusivamente como predadoras durante o período

Agência(s) de Financiamento: Embrapa Rondônia e CNPq 
T de pré-oviposição e continuam alimentando-se de seu hospedeiro mesmo depois de começar a fase de reprodução. Embora fêmeas de $C$. stephanoderis também se comportem como predadoras, alimentando-se de formas imaturas da broca do café, a oviposição ocorre preferencialmente na fase de pré-pupa e pupa da broca, possibilitando o desenvolvimento parasitário de sua prole sobre seu hospedeiro (LAUZière et al. 1999).

No Brasil, a introdução de C. stephanoderis para controle da broca-do-café ocorreu em meados da década de 1990 (Souza et al. 2006) e não há registros estatísticos de taxas do parasitismo desse parasitoide sobre a broca-do-café no país. No entanto, a eficiência deste inimigo natural tem sido comprovada a partir dos registros de outros países. No Togo, a taxas de mortalidade da broca, ocasionadas por C. stephanoderis, variaram entre $35 \mathrm{e}$ 48\% (Borbón 1991). Essa espécie de parasitoide foi introduzida em vários países da América Latina (CARBALLO 2002) e já se encontra estabelecida no Brasil, Equador, Honduras, Colômbia e México (Maldonado \& Benavides 2007). C. stephanoderis tem mostrado melhores resultados nas taxas de parasitismo de $H$. hampei que as demais espécies de parasitoides (INFANTE et al. 1994).

Como no Brasil não há registro de taxas do parasitismo de $C$. stephanoderis sobre população de H. hampei, este trabalho tem como objetivo, registrar a porcentagem de tal interação ecológica ocorrida no Estado de Rondônia em frutos presentes em dois ambientes: planta e solo.

\section{MATERIAL E MÉTODOS}

Este estudo foi realizado durante o ano 2004 no Campo Experimental da Embrapa-Rondônia, no município de Ouro Preto D'Oeste localizada a $10^{\circ} 45^{\prime}$ de latitude Sul e $62^{\circ} 15^{\prime}$ de longitude Oeste. A área consistiu de cultura de café Conilon (Coffea canephora Pierre ex A. Froehner), sem uso de agrotóxicos, com fileiras plantadas no espaçamento de 4,0 x 1,0 m. As plantas foram enumeradas para a realização de sorteio dos exemplares para a coleta dos frutos nos dias devidamente estipulados.

Durante o período de estudo, coletou-se 48 amostras nos meses de janeiro a dezembro. Em cada amostra, sortearam-se plantas para coleta de cinco frutos da parte aérea $(10 \times 5=50)$ e cinco frutos caídos sobre a superfície do solo $(10 \times 5=50)$, embaixo da planta, totalizando 100 frutos semanais. Durante todo o período amostral, coletaram-se 4.800 frutos secos do lote para as avaliações $(n=2.400 /$ planta $n=2.400 /$ solo $)$. Os frutos foram acondicionados em sacos de papel, etiquetados, e encaminhados ao Laboratório de Entomologia da Embrapa Rondônia, em Porto Velho, RO. No laboratório, os frutos foram dissecados e avaliados utilizando microscópio estereoscópio.

Os frutos dissecados com presença de formas imaturas de $H$. hampei e adultos vivos dentro dos frutos foram considerados frutos infestados. Para a quantificação percentual de frutos infestados, utilizou-se a equação proposta por ARISTIZÁbal et al. (1998), configurada da seguinte forma:

$$
N i=\left({ }^{F c i} /{ }_{N f d}\right) x 100
$$

$\mathrm{Ni}=$ níveis de infestação; $\mathrm{Fci}=$ frutos colonizados com formas imaturas de H. hampei; $N f d=$ número de frutos dissecados.
Os frutos dissecados com presença de formas imaturas de $H$. hampei e adultos vivos de C. stephanoderis foram considerados parasitados. A porcentagem de parasitismo foi calculada através da equação abaixo:

$$
T p p=\left({ }^{P f c} /{ }_{\text {Nfd }}\right) x 100
$$

$T p p=$ taxa percentual de parasitismo $P f c=$ número de frutos colonizados com presença do parasitoide; $N f d=$ número de frutos dissecados.

Para o cálculo do índice de parasitismo (IP) aplicou-se a equação proposta por SANTOS \& FreITAS (2008). A seguir:

$$
T p p=\left({ }^{P i} /{ }^{t}\right) x 100
$$

$P i=$ número de parasitoides obtidos de frutos para cada um dos ambientes; $P t=$ número total de parasitoides (nos dois ambientes investigados); $N o=$ número de frutos com formas imaturas de $H$. hampei (larvas e pupas) em cada ambiente.

Todos os parasitoide adultos coletados dos frutos de café foram acondicionados em frascos de vidro contendo álcool a $70 \%$, lacrados e etiquetados. Posteriormente, foram enviados para o Instituto Biológico, em São Paulo, para identificação do gênero taxonômico. Posteriormente, outro lote foi enviado para a Universidade Federal do Espírito Santo, Departamento de Biologia, onde foi identificada a espécie do parasitoide.

\section{RESULTADOS E DISCUSSÃO}

Porcentagem de parasitismo na planta e no solo. Verificou-se a presença do parasitoide $C$. stephanoderis em frutos brocados infestados com larvas e pupas de $H$. hampei. A porcentagem de parasitismo de C. stephanoderis, durante o período experimental, variou de 2 a $20 \%$ nas plantas e 2 a $24 \%$ no solo. O somatório das médias durante o período experimental (janeiro a dezembro) apresentou maior taxa de parasitismo na planta, sendo $62 \%$ nesse ambiente e $36 \%$ no solo (Figura 1). Nas plantas, observou-se 137 larvas e 94 pupas da broca-do-café nos frutos dissecados. No solo constatou-se 75 larvas e 51 pupas da broca-do-café. Dessa forma, constatou-se, maior porcentagem nas brocas presentes nos frutos coletados das plantas, devido à maior disponibilidade de hospedeiros (Tabela 1).

Esse resultado demonstra a especificidade e dependência do parasitoide $C$. stephanoderis em relação à disponibilidade de seu hospedeiro. Também estímulos químicos do hospedeiro estão envolvidos na localização do parasitoide, o que pode acarretar numa porcentagem mais alta de parasitismo no ambiente onde há alta densidade populacional da broca-do-café (CHIU-AlvarAdo et al 2010).

No período de colheita, normalmente os frutos encontram-se maduros o que é uma condição ótima para a broca-do-café se reproduzir e ovipositar. Isso disponibiliza maior quantidade de hospedeiros para o parasitoide. Portanto, nessa época observouse maior porcentagem de parasitismo, sendo $20 \%$ nas plantas (no mês de junho) e 24\% no solo (no mês de maio) (Figura 1).

Tabela 1. Infestação de diferentes estágios de H. hampei em frutos de C. canephora e índice de parasitismo por C. stephanoderis em um campo experimental da Embrapa localizado em Ouro Preto D’Oeste, RO, em 2004.

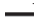

Ambientes

No de brocas adultas

No de frutos c/ ovos

No de frutos c/ larvas

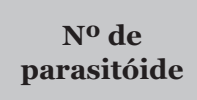

137

75

212

No de frutos
c/ pupas

94
51

94
51

145

195

195
65

260

$\%$
parasitismo

62

36 98
Índice de parasitismo (IP) 


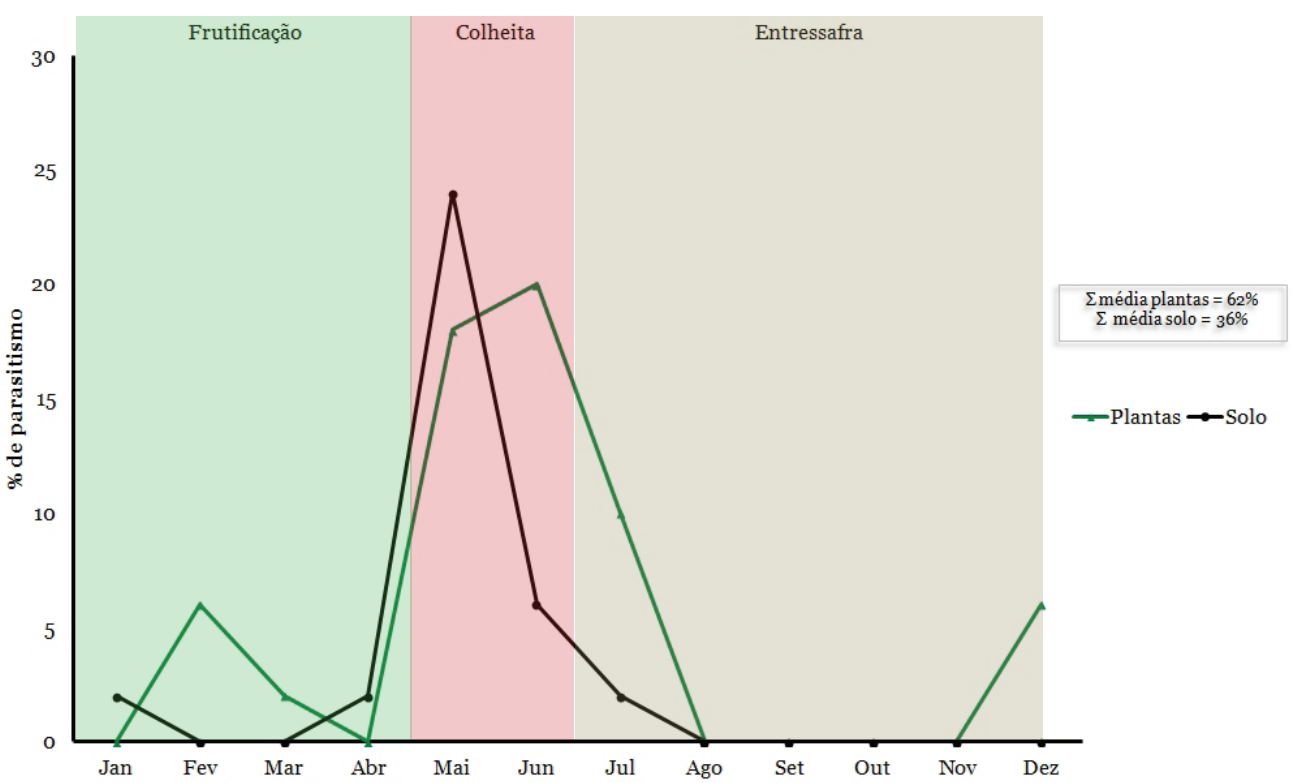

Figura 1. Parasitismo médio mensal de Cephalonomia stephanoderis sobre Hypothenemus hampei no período de janeiro a dezembro de 2004, nos ambientes planta e solo em área experimental da Embrapa Ouro Preto D’Oeste, RO.

Valores entre 3 e 10 parasitoides por fruto infestado no campo são indicadores do bom potencial de C. stephanoderis, o que ocasiona uma diminuição na taxa de infestação da broca. O nível de ataque pode variar entre $48 \%$ até $65 \%$ (PARDEY 2006). Na Colômbia, ARISTIZÁBAl et al. (1997) liberaram C. stephanoderis com níveis baixos de infestação de $H$. hampei e observaram taxas de parasitismo entre 5,3 e $26,2 \%$, oscilando entre 3,6 e $18,2 \%$ em frutos coletados nas plantas e entre o e $8 \%$ em frutos do solo. BenAvides et al. (1994) observaram taxas de parasitismo entre 22 e 65\% quando o nível de infestação de $H$. hampei foi maior que $80 \%$. Damon (2000) cita taxas de parasitismo de até $80 \%$ evidenciando o estabelecimento do parasitoide $C$. stephanoderis no México.

Índice de parasitismo na planta e no solo. O IP (Índice de Parasitismo) na área investigada foi de 217,5 nas plantas e 35,5 no solo (Tabela 1). Essa diferença, como se constatou na porcentagem de parasitismo, ocorre devido à presença de hospedeiros do parasitoide na planta ser mais acentuado em relação ao solo. Um total de 260 parasitoides foram encontrados nos dois ambientes estudados. Isso indica que o parasitoide encontra-se devidamente estabelecido nessa região de cultivo de café no Estado de Rondônia, dessa forma, efetuando parasitismo sobre a broca-do-café, seu hospedeiro específico (Tabela 1).

Relação da densidade populacional: hospedeiro e parasitoide. Foi feita a comparação da porcentagem de frutos infestados e a taxa de parasitismo nos dois ambientes investigados, durante a frutificação (janeiro a abril), colheita (maio e junho) e entressafra (julho a dezembro). As porcentagens de parasitismo acompanharam proporcionalmente as porcentagens de infestação do $H$. hampei nas plantas e no solo. No período da colheita, nas plantas observou-se $71 \%$ de frutos infestados e $26,3 \%$ de parasitismo; no solo constatou-se 55,7\% de frutos infestados e 17,3\% de parasitismo (Figura 2).

Naturalmente, a explicação para esse fenômeno evidencia a especificidade do parasitoide e demonstra sua dependência em relação ao hospedeiro para o ciclo reprodutivo e garantia de sua sobrevivência no ambiente. Portanto, a densidade populacional do parasitoide depende da densidade de seu hospedeiro, a brocado-café (Souza et al. 2006).

Quando um inimigo natural é especificamente dependente de sua presa, ou hospedeiro, como ocorre no caso do parasitoide

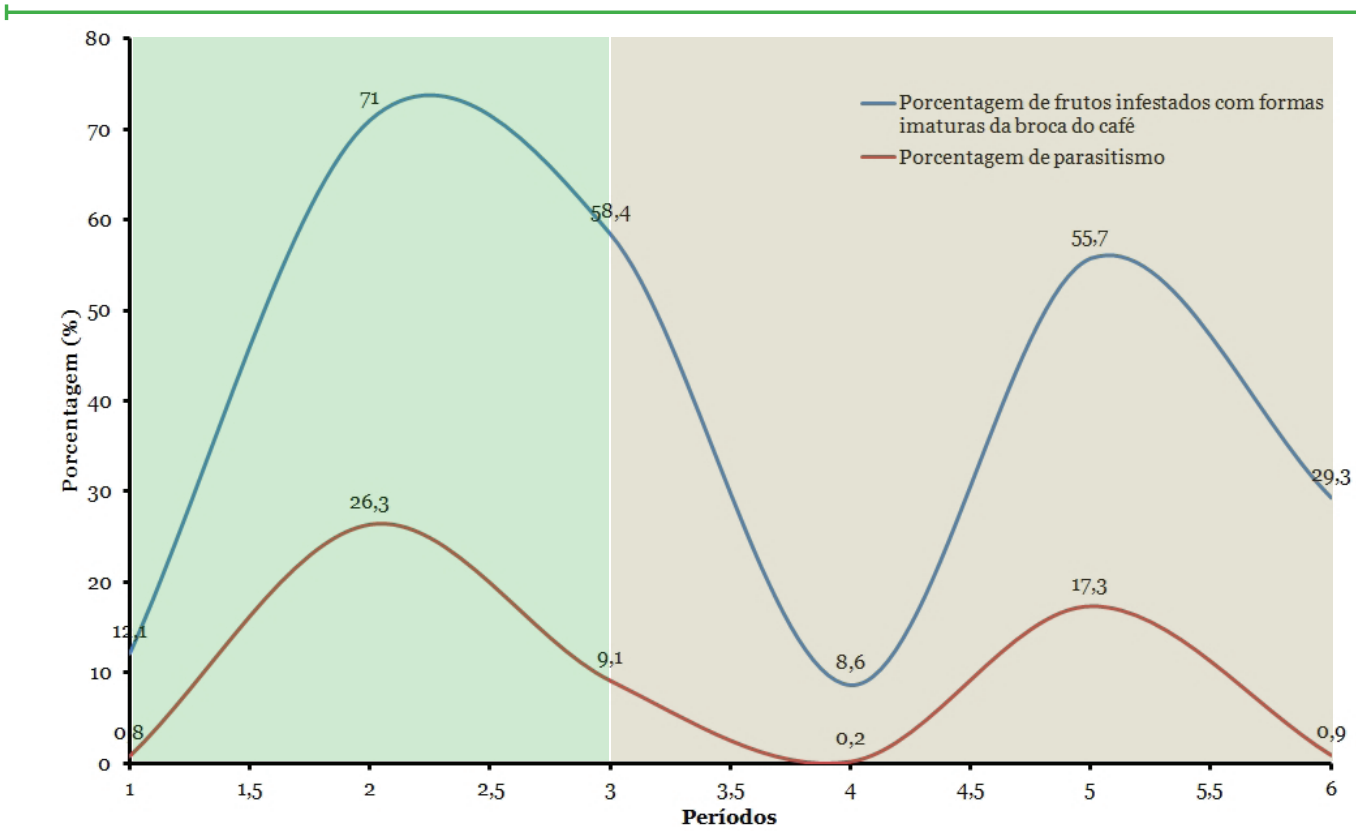

Figura 2. Média porcentual de frutos infestados e parasitismo por época: frutificação (jan. a abr.); colheita (mai. a jun.); entressafra (jul. a dez.) nos ambientes planta e solo. Os períodos 1, 2 e 3 representam respectivamente as épocas de frutificação, colheita e entressafra, nas plantas. Os períodos 4, 5 e 6 representam respectivamente as épocas de frutificação, colheita e entressafra, no solo. 
C. stephanoderis, a presença de H. hampei durante todo o ano no ambiente, é fundamental para a manutenção e equilíbrio populacional do inimigo natural. No entanto, observou-se que o fator determinante para a ocorrência do parasitoide no ambiente é a presença de formas imaturas da broca-do-café, que são os estágios escolhidos do parasitoide para sua alimentação e reprodução, corroborando com outros estudos a respeito deste parasitoide. Abraham \& Moore (1990) citam que a espécie $C$. stephanoderis é muito comum em lavouras cafeeiras na África, possivelmente, por atacar a maioria dos estágios imaturos do seu hospedeiro. LAUZIÈRE et al. (2001), constataram em laboratório, que o comportamento das fêmeas de $C$. stephanoderis envolve estratégias para explorar diferentes estágios de $H$. hampei, os quais são simultaneamente encontrados em frutos colonizados. Porém, conforme já mencionado neste artigo, apesar dessa espécie atacar todos os estágios de desenvolvimento da broca, sua oviposição ocorre preferencialmente em pré-pupa e pupas e em uma proporção menor em larvas do último instar (LAUZIÈRE et al. 1999; PÉrez-LACHAUd et al. 2004).

Por outro lado, estudos com outras espécies de parasitoides têm demonstrado a importância da densidade do hospedeiro na população de parasitoides (FoErster et al. 2001; GuILLoux et al. 2003; SANTOS \& Freitas 2008). Esses parâmetros ecológicos estão em conformidade com a maioria dos modelos analíticos, que predizem que é proporcional o aumento populacional do parasitoide com a disponibilidade de hospedeiros no meio ambiente (JERVIS \& KIDD 1986; KIDD \& JERVIS 1989; LAUZIÈRE et al. 1999). LAUZIÈRE et al. (2000), em ensaios de laboratório, constataram que a eficácia de $C$. stephanoderis como agente de controle biológico depende das variações da densidade de seu hospedeiro no ambiente.

GómEz et al.(2005), cita que um dos fatores que limita a efetividade de $C$. stephanoderis no campo é a baixa disponibilidade de $H$. hampei em estágios de desenvolvimento de sua vida suscetíveis de serem parasitados no período da entressafra. Dessa forma, constatou-se que após a alta disponibilidade de hospedeiros suscetíveis para a reprodução de C. stephanoderis, há uma tendência, depois de algumas semanas que novas gerações do parasitoide permaneçam no ambiente. Portanto, quando há uma diminuição na porcentagem de frutos colonizados com formas imaturas de $H$. hampei há, consequentemente, uma diminuição na densidade populacional do parasitoide $C$. stephanoderis nos dois ambientes investigados (Figura 2). Obviamente, isso acontece devido à escassez de hospedeiros, que é essencial para que o parasitoide possa completar seu ciclo biológico.

Isso torna evidente porque durante o período da colheita, o qual ocorre maior disponibilidade de formas imaturas de H. hampei, aumente a população do parasitoide neste período. Assim, nessa época é observada maior densidade populacional desse inseto. Apenas no final da entressafra o parasitoide torna a ressurgir no ambiente (Figura 2), época em que voltam a aparecer também formas imaturas do $H$. hampei nos frutos.

Provavelmente, os espécimes de parasitoides encontrados no campo experimental, são oriundos de frutos de outra região do Brasil, especificamente no Estado do Espírito Santo, onde já houve liberação desse inseto para o controle biológico de $H$. hampei (BENASSI \& BUSOLI 2007). Apesar de não ter ocorrido uma liberação massal desse parasitoide em Rondônia, observou-se a ação parasitária de $C$. stephanoderis sobre a população da brocado-café.

Este parasitóide encontra-se em um ambiente favorável para seu estabelecimento e reprodução, apresentando bons índices de parasitismo, o que o torna um promissor agente de controle sobre a população de $H$. hampei no município de Ouro Preto D’Oeste, em Rondônia.

C. stephanoderis é um importante agente de controle biológico que pode ser utilizado para o controle da broca-do-café em Rondônia e nas demais áreas de plantações de café no Brasil. Contudo, são necessários novos estudos são para viabilizar a criação massal do parasitoide em laboratório e liberação nas áreas de plantio do café.

\section{AGRADECIMENTOS}

Agradecemos à Embrapa Rondônia pelo apoio logístico que possibilitou o desenvolvimento da pesquisa. Ao CNPq pela bolsa concedida ao primeiro autor. Aos pesquisadores Valmir Antônio Costa do Instituto Biológico de São Paulo e Celso Oliveira Azevedo do Departamento de Biologia da Universidade Federal do Espírito Santo pela identificação taxonômica do parasitoide.

\section{REFERÊNCIAS}

Abraham, Y.J. \& D. Moore, 1990. Rearing and aspects of biology of Cephalonomia stephanoderis and Prorops nasuta (Hymenoptera: Bethylidae) parasitoids of the coffee berry borer, Hypothenemus hampei (Coleoptera: Scolytidae). Bulletin of Entomological Reserarch, 80: 120-128.

Aristizábal, A.L.F., P.A.E. Bustillo, H.J. Orozco \& C.B. Chaves, 1998. Efecto del parasitoide Cephalonomia stephanoderis (Hymenoptera: Bethyliade) sobre las poblaciones de Hypothenemus hampei (Coleoptera: Scolytidae) durante y después de la cosecha. Revista Colombiana de Entomología, 24: 149-155.

Aristizábal, A.L.F., P.S. Baker, H.J. Orozco \& C.B. Chaves, 1997. Parasitismo de Cephalonomia stephanoderis Betrem sobre una población de Hypothenemus hampei (Ferrari) con niveles bajos de infestación en campo. Revista Colombiana de Entomología, 23: 157-164.

Chiu-Alvarado P., J. Valle-Mora \& J.C. Rojas, 2010. Chemical cues from the coffee berry borer influence the locomotory behaviour of its bethylid parasitoids. Bulletin of Entomological Research, 6: 707-714.

Benassi, V. L. R. M. \& A. C. Busoli, 2007. Levantamento e índices de parasitismo de Cephalonomia stephanoderis BETREM (HYMENOPTERA: BETHYLIDAE), no norte do Espirito Santo. SPCB, 5: 2007, Águas de Lindóia, SP - Resumos expandidos (391), 5p. Disponível em: <http://www.sbicafe. ufv.br/handle/10820/1909>. [Acesso em: 05.11.2014].

Benavides M.P., P.A.E. Bustillo \& E. Montoya, 1994. Avances sobre el uso del parasitoide Cephalonomia stephanoderis para el control de la broca del café Hypothenemus hampei. Revista Colombiana de Entomologia, 20: 247 - 253.

Borbón O.M., 1991. La broca del fruto del cafeto: programa cooperativo ICAFE-MAG. $1^{\text {a }}$ ed. ICAFE. San José, Costa Rica. 50 p.

Carballo, M., 2002. Manejo Integrado de Plagas y Agroecologia. Costa Rica. 6: 118-122. Disponível em: <http://web.catie. ac.cr/informacion/RMIP/rev66/productos fitosan.pdf $>$. [Acesso em: 07.11.2013].

Costa, J.N.M., R.B. da Silva, P. de A. Ribeiro \& A. Garcia, 2002. Ocorrência de Beauveria bassiana (Bals.) Vuill. em brocado-café (Hypothenemus hampei, Ferrari) no Estado de Rondônia, Brasil. Acta Amazonica. 32:517-519.

Costa, J.N.M., P. de A. Ribeiro \& R.B. da Silva, 200o. Broca-docafé: previsão de infestação e recomendações de controle para a safra 2000/2001 no estado de Rondônia. RT/22, EMBRAPA-CPAF Rondônia, 2-5.

Damon, A. \& J. Valle, 2002. Comparison of two release techniques for the use of Cephalonomia stephanoderis (Hymenoptera: Bethylidae), to control the coffee berry borer Hypothenemus hampei (Coleoptera: Scolytidae) in Soconusco, southeastern Mexico. Biological Control, 24: 117-127.

Damon, A., 2000. A review of the biology and control of the coffee berry borer, Hypothenemus hampei (Coleoptera: Scolytidae). Bulletin of Entomological Research, 90: 453-465.

Foerster, L.A., A.K. Doetzer \& M.R.F. Avanci, 2001. Parasitoides larvais de Mythimna (Pseudaletia) sequax 
Franclemont e capacidade de parasitismo de Glyptapanteles muesebecki (Blanchard) em relação ao tempo de exposição, temperatura e densidade de hospedeiros. Acta Biológica Paranaense, 30: 139-149.

Gómez, J., J.F. Barrera, J.C. Rojas, J. Macias-Samano, J.P. Liedo, L. Cruz-Lopez \& M.H. Badii, 2005. Volatile Compounds Released by disturbed females of Cephalonomia stephanoderis (Hymenoptera: Bethylidae): a parasitoid of the coffee berry borer Hypothenemus hampei (Coleoptera: Scolytidae). Florida Entomologist, 88: 180-187.

Guilloux, T., R. Monnerat, M. Castelo Branco, A. Kirk \& D. Bordat, 2003. Population dynamics of Plutella xylostella (Lep., Yponomeutidae) and its parasitoids in the region of Brasilia. Journal of Applied Entomology. 127: 288-292.

Infante, F., J. Valdez, D.I. Penagos \& J.F. Barrera, 1994. Description of the life stages of Cephalonomia stephanoderis (Hymenoptera: Bethylidae) a parasitoid of Hypothenemus hampei (Coleoptera: Scolytidae). Vedalia, 1: 13-18.

Jervis, M.A. \& N.A.C. Kidd, 1986. Host-feeding strategies in hymenopteran parasitoid. Bioligical Reviews, 61: 395-434.

Kidd N.A.C. \& M.A. Jervis, 1989. The effects of host-feeding behaviour on the dynamics of parasitoid-host interactions, and the implications for biological control. Researches on Population Ecology, 31: 235-274.

Lachaud, G.P. \& I.C.W. Hardy, 2001. Alternative Hosts for Bethylid Parasitoids of the Coffee Berry Borer, Hypothenemus hampei (Coleoptera: Scolytidae). Biological Control, 22: 265277.

Laurentino, E. \& J.N.M. Costa, 2004. Descrição e caracterização biológica da broca-do-café (Hypothenemus hampei, Ferrari 1867) no Estado de Rondônia. Porto Velho: EMBRAPA Rondônia (Documentos, 90), 21p.

Lauzière, I., J. Brodeur \& G. Pérez-Lachaud, 2001. Host stage selection and suitability in Cephalonomia stephanoderis Betrem (Hymenoptera: Bethylidae), a parasitoid of the coffee berry borer. Biological Control, 21: 128-133.

Lauzière, I., G. Pérez-Lauchaud \& J. Brodeur, 2000. Behavior and Activity Pattern of Cephalonomia stephanoderis (Hymenoptera: Bethylidae) Attacking the Coffee Berry Borer, Hypothenemus hampei(Coleoptera: Scolytidae). Journal of Insect Behavior, 13: 375-395.

Lauzière, I., G. Pérez-Lauchaud \& J. Brodeur, 1999. Influence of host density on the reproductive strategy of Cephalonomia stephanoderis, a parasitoid of the coffee berry borer. Entomologia Experimentalis et Applicata, 92: 21-28.
Maldonado L., C.E. \& P. Benavides-Machado, 2007. Evaluación del establecimiento de Cephalonomia stephanoderis y Prorops nasuta, controladores de Hypothenemus hampei, en Colombia. Cenicafé, 58: 333-339.

Pardey, A.E.B., 2006. Una revisión sobre la broca del café, Hypothenemus hampei (Coleoptera: Curculionidae: Scolytinae), en Colombia. Revista Colombiana de Entomología, 32: 101-116.

Pérez-Lachaud, G., T.P. Batchelor \& I.C.W. Hardy, 2004. Wasp eat wasp: facultative hyperparasitism and intra-guild predation by bethylid wasps. Biological Control, 30: 149-155.

Pérez-Lachaud, G., I.C.W. Hardy \& J.P. Lachaud, 2002. Insect gladiators: competitive interactions between three species of bethylid wasps attacking the coffee berry borer, Hypothenemus hampei (Coleoptera: Scolytidae). Biological Control, 25: 231-238.

Reis, P.R., J.C. Souza \& M. Venzon, 2002. Manejo ecológico das principais pragas do cafeeiro. Informe Agropecuário, 23: 8399.

Santos, R.S. \& S. Freitas, 2008. Parasitismo de Erythmelus tingitiphagus (Soares) (Hymenoptera: Mymaridae) em Ovos de Leptopharsa heveae Drake \& Poor (Hemiptera: Tingidae), em Plantios de Seringueira (Hevea brasiliensis Müell. Arg.). Neotropical Entomology, 37: 571-576.

Salazar, H.M. \& P.S. Baker, 2002. Impacto de liberaciones de Cephalonomia stephanoderis sobre poblaciones de Hypothenemus hampei. Revista Cenicafé, 53: 306-316.

Souza, M.S, C.A.D. Teixeira, C.O. Azevedo, V.A. Costa \& J.N.M. Costa, 2006. Ocorrência de Cephalonomia stephanoderis Betrem (Hymenoptera: Bethylidae) em Cafezais da Amazônia Brasileira. Neotropical Entomology, 35: 560-562.

Vega, F.E., F. Infante, A. Castillo \& J. Jaramillo, 2009. The coffee berry borer, Hypothenemus hampei (Ferrari) (Coleoptera:Curculionidae): a short review, with recent findings and future research directions. Terrestrial Arthropod Reviews, 2: 129-147.

\section{Recebido em: o8/11/2013 \\ Aceito em: 04/o6/2014}

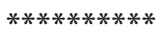

\section{Como citar este artigo:}

Souza, M.S., A.A. Silva, C.A.D. Teixeira \& J.N.M. Costa, 2014. Parasitismo na população da broca-do-café Hypothenemus hampei (Ferrari) (Coleoptera: Curculionidae), pelo parasitoide Cephalonomia stephanoderis Betrem (Hymenoptera: Bethylidae). EntomoBrasilis, 7 (3): 178-182. Acessível em: doi:10.12741/ebrasilis.v7i3.402
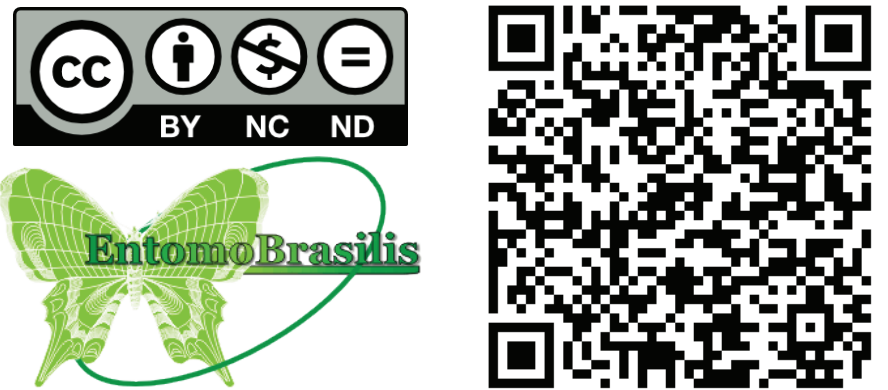